\title{
TV/Series
}

\section{Délibération et circulation des idées entre l'Aile ouest et la Situation Room}

\section{Perig Pitrou}

\section{(2) OpenEdition}

1 Journals

Édition électronique

URL : http://journals.openedition.org/tvseries/668

DOI : $10.4000 /$ tvseries.668

ISSN : 2266-0909

Éditeur

GRIC - Groupe de recherche Identités et Cultures

Référence électronique

Perig Pitrou, «Délibération et circulation des idées entre l'Aile ouest et la Situation Room », TV/Series [En ligne], 8| 2015, mis en ligne le 01 décembre 2015, consulté le 19 avril 2019. URL : http:// journals.openedition.org/tvseries/668; DOI : 10.4000/tvseries.668

Ce document a été généré automatiquement le 19 avril 2019

\section{(c) (i) (2) $\Theta$}

TV/Series est mis à disposition selon les termes de la licence Creative Commons Attribution - Pas d'Utilisation Commerciale - Pas de Modification 4.0 International. 


\title{
Délibération et circulation des idées entre l'Aile ouest et la Situation Room
}

\section{Perig Pitrou}

\author{
« Take a look at this book. This is exciting stuff. \\ It's about who we are and what we want » \\ Le Président à propos du livre de la Constitution \\ des États-Unis qu'il offre à sa fille. \\ The American President (Aaron Sorkin, 1995)
}

1 Avant d'étudier certaines thématiques liées à The West Wing, je commencerai par faire un court détour par le Mexique, pays dont ses habitants n'hésitent pas à déplorer avec humour qu'il est « si loin de Dieu et si proche des États-Unis » (tan lejo de Dios y tan cerca de los Estados Unidos). Lors d'une enquête ethnographique de deux ans réalisée chez les Mixe ${ }^{1}$ de l'État de Oaxaca au Mexique, j'ai été amené à accompagner les représentants d'un village lors des rites d'intronisation. Dans les communautés villageoises paysannes où vivent les 130000 Mixe, les habitants se sont vu accorder une autonomie par le pouvoir central, qui les autorise à élire leurs représentants lors d'assemblées générales, sans passer par un système de vote à bulletins secrets. Chaque année, un groupe d'hommes est ainsi tenu d'assumer bénévolement une charge à la suite d'une cérémonie lors de laquelle les membres de l'équipe municipale sortante remettent des bâtons de commandement à leurs successeurs sur la place du village ; cette présentation publique est complétée par des parcours rituels, plus secrets, lors desquels les édiles pratiquent nuitamment des sacrifices de volailles dans la mairie. À cette occasion, ils sollicitent l'intervention d'une entité appelée «Celui qui fait vivre » (yikjujyky'äjtpi) afin qu'elle les aide à remplir leur mission dans de bonnes conditions. Outre la protection, le maire et ses assistants sollicitent que cette entité « envoie des idées » ou « des pensées » ou aide à «trouver les bonnes paroles ", c'est-à-dire qu'elle participe aux processus de résolution de conflits : en plus de ses pouvoirs matériels, il se voit donc attribuer une capacité d'intervenir favorablement dans des activités cognitives et délibératives. Même si, à certains égards, l'«envoi de pensées » relève d'une forme d'hétéronomie, le dialogue et la réflexion collective sont hautement valorisés comme le prouvent les énoncés dans lesquels les énonciateurs déclarent espérer « trouver entre eux les idées ». 
2 Je ne chercherai pas à entrer davantage dans les détails concernant les conceptions autochtones du pouvoir, me permettant de renvoyer à d'autres travaux que j'ai consacrés à cette question, en particulier pour élucider la manière dont l'agentivité des nonhumains s'insère dans l'institution politico-judiciaire de résolution des conflits ${ }^{2}$. Je soulignerai seulement le parallèle qui m'est souvent venu à l'esprit entre les dispositifs mésoaméricains de légitimation du pouvoir, et les scènes se déroulant dans la Situation Room où certains protagonistes de The West Wing se retrouvent pour prendre des décisions cruciales. À l'instar de ce qui s'observe chez les Mixe, tout se passe comme si les délibérations relatives à la résolution de conflits nécessitaient la formation d'un pli ou d'un repli à l'intérieur de la Maison-Blanche, filmée en général comme un espace relativement ouvert et dans lequel les personnes, les paroles et les idées circulent avec une fluidité que tous les commentateurs ont remarquée (cf. l'article d'Ophir Lévy dans ce volume). C'est à partir d'un tel contraste que je souhaite aborder la problématique de la délibération qui constitue une des principales trames scénaristiques de la série : dans tous les épisodes, l'enjeu est en effet de suivre comment les idées élaborées par les différents conseillers sont transmises au Président Bartlet de façon à ce qu'il prenne les décisions adéquates. Étant coextensive à l'intégralité des épisodes, cette problématique ne saurait être traitée de façon exhaustive dans le cadre d'un article, mais l'examen de quelques scènes se déroulant dans la Situation Room, ou dans son orbe, permettra d'aborder des questions fondamentales aussi bien pour la série que pour une réflexion plus large relevant de l'anthropologie politique.

3 Hiérarchiquement, le Président est celui qui décide, mais la multiplicité des conseillers soulève le problème des limites de leur influence - ou, inversement du degré réel d'autonomie du «Leader of the free world». On se confronte donc ici à une question qui ne cesse de se reformuler depuis Les Lois, en passant par Le Prince, jusqu'aux débats relatifs au rôle des «spin doctors ": lorsque s'accroit le rôle du conseiller, qui est véritablement l'auteur des décisions prises par le gouvernant, que celles-ci s'expriment verbalement ou qu'elles s'inscrivent dans une signature? Ce n'est évidemment pas cette question, trop générale, que je traiterai en tant que telle ici. Mon intention est de faire apparaître les caractéristiques distinctives des décisions prises dans la salle de crise, ce qui implique de mettre au jour les dispositifs qui encadrent la circulation des idées lorsque la sécurité nationale ou internationale est menacée ainsi que les principes sur lesquels les délibérations s'appuient en ces occasions.

\section{La circulation des idées : fluidités et médiations}

4 Une des marques de fabrique de The West Wing est constituée par les scènes filmées en travelling durant lesquelles le spectateur suit les déplacements rapides des personnages, alors même qu'ils sont engagés dans des dialogues aussi fluides que précis, en dépit de leur haute technicité. C'est qu'il faut aller vite. Dans le cas le plus paradigmatique, la fin du parcours dans les couloirs correspond avec le moment où les conseillers entrent à l'intérieur du Bureau ovale: il convient alors d'être bref et de formuler clairement des propositions aidant le Président dans les innombrables décisions qu'il doit prendre quotidiennement. Les déplacements dans l'espace, indissociables d'un emploi du temps extrêmement chargé, rendent sensible une organisation hiérarchique exigeant que les idées les plus pertinentes convergent vers POTUS (President of the United States) afin qu'il fasse les choix les meilleurs pour l'avenir de la nation. 
Cela implique, comme Leo - le chef de cabinet de la Maison-Blanche - le recommande souvent aux conseillers, qu'une forme de stabilisation de l'hésitation ou, à tout le moins, qu'une réduction du nombre des options possibles soit réalisée au préalable; tout doit être mis en œuvre pour éviter que le Bureau ovale ne devienne un lieu de tension ou de dissensus, comme le montre bien l'épisode dans lequel Josh (chef de cabinet adjoint), souffrant d'un syndrome post-traumatique, ose élever la voix face au Président (2.10). Avant d'en arriver à cette étape finale de résolution - qui, dans les cas les plus épineux, présente ses méandres propres - lors de laquelle le Président tranche à partir des informations transmises, ses conseillers doivent eux-mêmes s'être engagés, individuellement ou collectivement, dans une activité délibérative qui les conduit à filtrer parmi toutes les informations qu'ils reçoivent celles qui sont vraiment pertinentes. Même si aucun des personnages n'échappe tout à fait, ne serait-ce que de manière subreptice, à l'hubris de la toute-puissance, c'est évidemment le contraire que les auteurs de The West Wing cherchent à mettre en valeur, c'est-à-dire un univers politique dans lequel les problèmes qui émanent de la société civile émergent progressivement dans le débat public jusqu'à être pris en compte par ceux qui sont aux responsabilités.

Dans sa préface à l'ouvrage Le Fantôme de l'esprit public de Walter Lippman ${ }^{3}$, Bruno Latour met en évidence avec beaucoup de perspicacité la façon dont des idées deviennent des problèmes (issues), à mesure qu'un individu ou un groupe d'individus parvient à élargir le cercle de ceux prêts à se mobiliser pour défendre une cause. Conformément à un phénomène que l'anthropologie politique a souvent observé dans les organisations hiérarchiques, le bureau de chacun des conseillers fonctionne comme une « réplique » du Bureau ovale, c'est-à-dire comme un lieu de convergence, vers lequel des visiteurs viennent régulièrement faire " remonter », comme on dit, des revendications ou défendre des projets qui leur tiennent à cœur. Que ce soit lors des discussions à bâtons rompus qui accompagnent des déplacements ou des entretiens dans des bureaux, The West Wing ne cesse donc jamais de faire l'éloge de la fonction résolutive et consensuelle de la parole en acte.

7 Après les pouvoirs de la plaidoirie auxquels le cinéma américain nous a accoutumés - par exemple dans Twelve Angry Men (1957) de Sidney Lumet, ou dans Mr Smith Goes to Washington (1939) de Franck Capra - on voit ici représentée la façon dont un collectif parvient à construire une décision juste, à partir de la réitération de séquences dialogiques. Même si nous sommes invités à voir les choses de l'intérieur, ce n'est donc pas tant l'idéal de la transparence qui constitue l'horizon de ce processus que celui d'une connexion démocratique unissant le Président à son peuple sans solution de continuité.

8 La multiplicité des conseillers - surtout si l'on tient compte de ceux qui n'apparaissent pas dans l'organigramme officiel - introduit cependant de la différence: chacun intervient en fonction de principes spécifiques qui orientent sa perception du réel. L'impression de continuité produite par les scènes de travelling ne doit pas en effet faire oublier que les délibérations ou les accords sont toujours le fruit d'un intense travail fourni par les divers acteurs pour combiner de l'hétérogène ; l'intérêt de la fiction tenant précisément dans sa capacité à incarner les différences dans des personnages dont les compétences ou la sensibilité ouvrent sur une pluralité de perspectives sur le monde. Dans De la critique $e^{4}$, Boltanski propose de distinguer le «monde» et la "réalité »: le premier correspondant à «l'ensemble de ce qui arrive» - pour reprendre la formule de Wittgenstein - tandis la seconde désigne ce qui, dans une construction sociale donnée, semble normal aux acteurs qui y évoluent. Similairement, on pourrait soutenir que dans 
The West Wing chaque personnage fait transiter des informations, à partir d'une ponction sélective de vues sur le monde, qui offrent une version différentielle du réel construite avant d'être proposée au Président. Face à une telle dissémination, la continuité dans la circulation des idées ne s'obtient qu'au prix d'importants efforts pour surmonter des discontinuités et assembler des positions dissemblables, voire antagonistes.

L'enquête sur les modes d'existence ${ }^{5}$ de Latour contient des instruments analytiques et descriptifs fort utiles lorsqu'on cherche à suivre la circulation des idées dans des réseaux à l'intérieur desquels s'enchevêtrent et s'agencent les humains et les non-humains tels que des machines, des dieux ou des objets. Similairement, dans The West Wing, la mise en images exige de maintenir dans l'existence tous ces êtres en les insérant dans un réseau cohérent ce qui apparente le travail du scénariste à celui de l'ethnographe : l'un comme l'autre doivent être capables de repérer les objets qui sont vraiment indispensables au bon déroulement des interactions. Des pages entières pourraient ainsi inventorier la multitude d'objets ${ }^{6}$ qui participent peu ou prou, à l'élaboration des informations : les écrans (téléviseur, ordinateur, vidéoconférence), les téléphones, les petites notes, les bureaux, les stylos, les micros, tous participent au transfert des idées entre les personnages et d'un lieu à un autre.

10 Certains objets renvoient aux procédés technico-cognitifs spécifiques qui caractérisent les compétences propres à certains personnages lorsqu'ils réfléchissent. La capacité de Josh à avoir sans cesse présent à l'esprit l'équilibre des forces au Congrès se manifeste dans un tableau à fiches placé dans son bureau. L'accès à des informations stratégiques inhérent à la position de Leo explique qu'il manipule souvent des petites notes en papier ou un téléphone qui le connectent avec des interlocuteurs inconnus. Toby, le directeur de la communication, pour des raisons analysées plus loin, manipule souvent des livres de droit et, conformément à sa fonction de rédacteur de discours, n'est jamais très éloigné de son carnet de notes. On ajoutera d'ailleurs que ce dernier objet indique que les délibérations du Président ne sont pas seulement orientées par les informations transmises : elles s'énoncent aussi à partir des mots mis à sa disposition par ceux qui ont pensé pour lui.

11 Il serait possible de continuer à inventorier la spécificité discursive et technique du décryptage du monde auquel procèdent des protagonistes tels que Sam (directeur-adjoint de la communication), C.J. (la porte-parole de la Maison-Blanche), Charlie (assistant personnel du Président, mais aussi Joey Lucas, la spécialiste des sondages, les militaires, voire même le psychanalyste ou le prêtre qui apparaissent dans certains épisodes. L'important est de noter que ces procédures s'effectuent en mobilisant des principes généraux censés orienter l'action individuelle et collective. Dans la plupart des épisodes, la pluralité, synonyme de divergence ou de désaccord, ne fait pas obstacle à un travail d'unification et d'agencement des positions des uns et des autres, même si cela occasionne parfois des tensions ou des frustrations.

12 En revanche, dans d'autres épisodes, le conflit entre les principes mobilisés par les acteurs devient si intense que la dimension dramatique de l'exercice du pouvoir se fait jour. Ces moments de tensions, qui obligent les participants à produire des «justifications» (Boltanski \& Thévenot $\left.{ }^{7}\right)$, c'est-à-dire à expliciter et à défendre les principes à l'aune desquels ils veulent juger les situations, constituent un terrain privilégié pour étudier les apories dont Bartlet cherche à sortir. Dans ce cadre, le conflit rémanent entre pouvoir civil et pouvoir militaire possède une valeur paradigmatique, c'est pourquoi il convient de jeter un œil sur ce qui se déroule dans la Situation Room, un 
espace dont le fonctionnement constitue une sorte d'opposé structural à celui observé dans l'Aile ouest.

Les contrastes que les scènes filmées dans la salle de crise introduisent à l'intérieur d'une organisation fluide sont si importants qu'il n'est pas exagéré d'affirmer qu'à plus d'un titre, l'entrée dans cet univers produit une sorte de changement de régime. C'est tout d'abord les modalités de la circulation des personnes qui se modifient: alors que les bureaux sont la plupart du temps ouverts et que même les participants des visites organisées ont le droit d'accéder au Bureau ovale, parmi l'équipe du Président, seul lui et Leo - et C.J. lorsqu'elle lui succède - ont accès à cet espace sécurisé. Ce centre de gravité, creusé dans les fondements de l'édifice, auquel on accède par un déplacement vertical tend à inverser le mouvement de convergence, essentiellement horizontal, décrit précédemment. Même si les militaires et spécialistes du renseignement qui attendent assis à la table des opérations doivent se lever et être au garde-à-vous lorsque le "Commandant en chef» entre dans la pièce, cela ne doit pas faire perdre de vue que, dans ce contexte, c'est toujours le Président qui doit se déplacer lorsqu'on l'appelle. D'emblée, on comprend que le caractère exceptionnel de la Situation Room ne provient pas seulement de l'urgence des conflits: il dépend directement d'un équilibre spécial qui s'établit entre les instances du pouvoir.

Si l'influence des conseillers conduit parfois à s'interroger sur l'autonomie du Président, lorsqu'il est entouré par les militaires, les choses vont encore plus loin, et on a souvent l'impression que le représentant du pouvoir exécutif est subrepticement dépossédé de ses prérogatives au moment même où sa fonction de décideur atteint son paroxysme. Certes, en proposant à Bartlet des «scénarios" et en attendant qu'il donne des ordres, les militaires ne contestent jamais ouvertement la hiérarchie du commandement. Il n'en demeure pas moins que, dès lors que la question de l'usage de la force se pose, l'hésitation accompagnant d'ordinaire la délibération se mue en une sorte de flottement plus inquiétant concernant l'identité du véritable auteur des décisions prises. Ici - c'est-à-dire, aussi, dès qu'il est question de guerre - la discrétion et l'obéissance de Leo laissent place à une attitude plus affirmative, d'autant plus troublante qu'elle se manifeste au travers d'une sorte de discrète manipulation. Même quand Leo n'énonce pas explicitement la solution qui lui semble la meilleure, il n'est pas rare que Bartlet tourne vers lui des regards inquiets, comme s'il était possesseur d'un savoir secret.

En vérité, dans ces moments-là, alors que semble vaciller l'assurance de Jed Bartlet, on découvre que ce n'est rien moins que la fonction présidentielle qui traverse une épreuve à chaque descente dans la salle de crise. La première plongée dans cette réalité, lors de l'épisode "A proportional response » (« Riposte proportionnelle», 1.3), invite à ne pas faire un contresens : le problème ne saurait se résumer à l'opposition caricaturale entre un pouvoir civil pacifiste et des militaires prompts à entrer en guerre. Dans cet épisode, ce sont en effet les militaires, assistés par Leo, qui doivent contrôler la soif de vengeance de Bartlet et calmer son désir d'engager une réponse disproportionnée à l'attentat dont sont victimes les États-Unis : ce n'est donc pas tant la pusillanimité du Président - que Leo critique souvent - que son manque d'expérience dans l'art de la guerre et du champ de bataille qui le place en porte-à-faux vis-à-vis des militaires. Au contraire, Leo possède cette expérience, comme le prouve le très bel épisode dans lequel les flashbacks de son propre sauvetage lors de la guerre de Corée se superposent avec les séquences lors desquelles s'organise l'exfiltration de deux soldats depuis ce qui devient véritablement une " salle des opérations », autre traduction possible de Situation Room (5.14). 
16 En première analyse on peut affirmer que, paradoxalement, dans un endroit tout entier construit pour que les décisions soient prises par le Commandant des armées, le manque d'expérience de Bartlet le rend moins apte à imposer ses choix que les autres personnes assises autour de la table, situation singulière qui s'accompagne d'une modification du régime de visibilité et des procédures de captation du réel ainsi que de la temporalité qui en dépend. Il ne s'agit plus ici de suivre les innombrables cercles de la politique, les lentes constructions grâce auxquelles les idées de la multitude convergent vers la MaisonBlanche: au contraire, grâce aux vidéoconférences et aux images satellitaires, un mouvement centrifuge permet aux militaires d'étendre leur pouvoir jusqu'aux extrêmes limites du monde. Dans ce lieu clos et sans fenêtre, l'enjeu est alors de s'approcher, autant que possible, d'une forme de simultanéité de la perception tout autant que de l'action : à la différence des autres décisions du Président, celles prises dans cette salle des opérations sont immédiatement suivie d'effets. On trouve un très bon exemple de cette simultanéité dans l'épisode The War at Home (2.14) : alors même que Bartlet ordonne en temps réel aux forces spéciales de donner l'assaut à des cartels de la drogue en Colombie, il est immédiatement informé qu'un missile sol-air a touché un hélicoptère de l'armée tuant plusieurs militaires.

Dans ces contextes, on assiste à la transformation des modalités de l'expression des décisions présidentielles en se déplaçant du Bureau ovale vers la Situation Room. En haut, le pouvoir présidentiel se manifeste et s'incarne dans une signature tandis qu'en bas, c'est avec des signes beaucoup plus fugaces, par la parole, voire par un simple signe de la tête, que Bartlet lance les opérations militaires. Derrière le contraste entre deux espaces, c'est donc un antagonisme plus ou moins latent entre deux formes de pouvoir qui émerge : la première repose sur la force du droit et la légitimité démocratique, la seconde trouve son origine dans l'expérience de la guerre que les spécialistes de l'usage rationnel de la force se transmettent génération après génération.

18 C'est d'ailleurs en raison de cette transmission diachronique qu'on peut identifier un régime d'historicité spécifique à la salle des opérations. Par-delà le reenactment de l'expérience individuelle du champ de bataille que favorise le dispositif technico-sensoriel de la salle, on découvre que certains événements collectifs structurent les principes à partir desquels les militaires déploient leur interprétation du monde - et donc sa transformation. On en trouve un excellent exemple lorsque Bartlet et les militaires cherchent une solution pour arrêter Shareef, le Ministre de la Défense du Qumar, dont les liens avec les terroristes sont avérés. Comme la référence à l'élimination d'un général japonais durant la Seconde Guerre Mondiale l'indique dans le titre de l'épisode ( $W e$ Killed Yamamoto", "On a tué Yamamoto », 3.21), pour les militaires, la décision d'avoir recours à la force s'élabore à partir d'une mémoire spécifique du passé sur laquelle le recours à l'action vient s'appuyer.

Une fois repéré cet antagonisme, il convient d'approfondir l'analyse afin de ne pas se contenter de constater que les contrastes entre les espaces, les personnes ou les systèmes axiologiques renvoient à une opposition entre la force et le droit. Je concentrerai donc mon attention sur le déroulement des épisodes durant lesquels les problèmes liés à la mise à mort de Shareef occupent le devant de la scène (pour le spectateur), tout en étant traités dans un clair-obscur porteur d'une haute intensité dramatique. 


\section{L'elliptique du pouvoir et la construction mythique de la délibération}

20 Les auteurs de The West Wing sont trop subtils pour se limiter à opposer militaires et représentants élus. L'enjeu, tant pour la dramaturgie que pour la réflexion philosophique, est de déterminer comment ceux qui représentent ces deux instances de pouvoir en arrivent, concrètement, à prendre des décisions «ensemble ", étant entendu que cette forme d'association s'apparente souvent à une chimère au fonctionnement toujours instable. Cela ne signifie pas que le recours à la force serait une fois pour toute codifié et légitimé : au contraire, comme en attestent les âpres discussions provoquées par la découverte de l'implication de Shareef dans des activités terroristes, le travail par lequel un « attachement » (Latour) du droit et de la force est obtenu doit sans cesse être repris.

Bien qu'au début de l'épisode 3.20, les spécialistes militaires exposent des preuves irréfutables de la culpabilité de Shareef, Bartlet considère qu'elles sont insuffisantes pour être transmises au Ministère de la Justice. Comme il le répète à plusieurs reprises, son intention est de traîner Shareef devant les tribunaux et, dans cette perspective, il est indispensable de s'appuyer sur des éléments susceptibles d'être retenus comme valables dans le régime spécifique de véridiction propre à cet univers. Si les militaires considèrent détenir suffisamment d'informations, au regard de la justice, qui exige une requalification conformément au droit, on est encore loin du compte - nouvelle illustration de la pluralité des principes sur lesquels le travail de construction de l'information se développe, et Bartlet sort de la salle crise en déclarant: "You haven't got it ». Lors d'une autre réunion, on comprend cependant que le problème n'est pas seulement quantitatif et qu'un supplément d'information ne suffit pas.

Comme Latour l'établit bien dans La Fabrique du droit ${ }^{8}$, le fonctionnement des institutions chargées d'appliquer le droit exigent que toutes les décisions soient prises en conformité avec un ensemble de règles qu'il convient de ne pas oublier et de ne pas transgresser. Alors que les prévisions des militaires s'appuient sur l'analyse des situations passées, les délibérations des juristes sont liées à une chaine de décisions préalables qui ont été objectivées dans des textes de lois et, en dernière instance, toute décision se doit de ne pas contrevenir à la Constitution - dont le poids est particulièrement important aux États-Unis - ou à des principes fondamentaux liés aux droits de l'Homme. En dépit de la production de nouvelles preuves accablantes pour Shareef, Bartlet s'entend ainsi dire : " The judge would throw out the case. The entire chain of evidence leading us to Shareef originates with the testimony of a Chechnyan prisoner ». L'usage de la torture sur ce témoin - dans un pays l'autorisant - étant jugé illégal par la justice américaine, c'est donc l'ensemble de l'échafaudage judiciaire qui s'écroule, laissant Bartlet désemparé : jusqu'à la fin de cette opération, il ne cessera de déclarer son désir de faire juger le coupable ( I I want him tried ») plutôt que d'avoir recours à la force pour l'éliminer.

23 Face à cette impasse, une brèche dans la chaîne de commandement s'ouvre au moment où Leo reste dans la Situation Room, sachant que Fitzwallace souhaite, en marge des réunions, lui exposer son interprétation de la situation. La discussion s'engage à partir d'une question que le Général - ou les scénaristes - semble directement extraire du chapitre XIII du Léviathan ${ }^{9}$ : "Can you tell when it's peacetime and wartime anymore?». Leo a beau défendre la position du droit et des lois internationales qui protègent depuis l'Antiquité les diplomates ou déclarer qu'il n'apprécie guère la teneur de cette conversation, on sent 
bien qu'il le fait avec nettement moins de vigueur que Bartlet quelques minutes auparavant. Il faut dire que le Général Fitzwallace déploie une argumentation rigoureuse pour défendre la conception qu'il se fait du monde qui tient finalement en la prémisse suivante : on est en état de guerre. S'appuyant sur l'idée que même les lois de la nature ne s'appliquent pas - autre référence probable à Hobbes -, le militaire affirme clairement que le régime de véridiction dont relèvent certaines opérations militaires est irréductible à celui du droit.

24 À la fin de cet entretien, Leo semble convaincu, même s'il n'en dit rien ouvertement; il va d'ailleurs à son tour s'employer à faire changer Bartlet d'avis. À ce moment-là, la nature double - duplice? - de Leo se manifeste d'une façon flagrante : malgré son dévouement pour le Président, son passé de héros de guerre tout comme ses accointances avec le monde des militaires conduisent à faire souvent douter de sa neutralité. En nous le montrant discuter seul avec les gradés dans la salle de crise, le spectateur observe avec perplexité comment un personnage attachant se révèle également être le détenteur d'un pouvoir exorbitant alors même qu'il n'a pas été élu.

Dans les épisodes liés à l'affaire Shareef, l'ascendant, d'ordinaire modéré, de Leo sur son supérieur hiérarchique apparaît avec une certaine brutalité, comme la discussion qui s'engage dans le Bureau ovale en offre une parfaite illustration à la fin de l'épisode. Tout comme l'échange entre Leo et le Général qu'il duplique en le modifiant, la teneur de ce dialogue est hautement philosophique, en particulier lorsque Bartlet réitère sa volonté de ne pas transgresser les lois internationales et soutient - contre Leo qui lui reproche sa position idéaliste - qu'il existe des "absolus moraux" ("The are moral absolutes»). De telles déclarations ne manquent pas de panache et de fermeté, elles ne font cependant pas oublier que Bartlet les énonce au moment même où il semble abdiquer devant Leo emporté, en cette occasion, très loin par sa conviction, comme le prouvent les paroles qui passent la barrière de ses dents - pour reprendre l'expression d'Homère :

LEO. This is justified. This is required.

BARTLET. Says who?

LEO. Says me, Mr. President. You want to go ask some more people, they'll say so,

too.

Dans un épisode extrêmement réussi (5.8), les conseils de Josh permettent au Président de reprendre la main dans la lutte pour le leadership qui l'oppose au Republican Speaker of the House. Ici le spectateur devient témoin d'un conflit d'autant plus grave et inquiétant qu'il se déroule dans les interstices du fonctionnement des institutions tel qu'il est prévu par la Constitution. La référence de Leo à l'opinion des autres, censée valider la position défendue, confirme cette analyse : déclarer qu'on aurait le peuple derrière soi si on lui demandait son avis sera toujours différent d'en être un représentant élu devant rendre compte de ses actes. Pourtant Leo ne devient pas véritablement le détenteur du pouvoir, pas plus que la démocratie américaine ne se transforme en une dictature lorsque des actes aussi problématiques que l'assassinat d'un ennemi sont décidés.

Dans l'épisode qui clôture la troisième saison, fort judicieusement intitulé Posse Comitatus (« Assassinat politique », 3.22) - un principe politique selon lequel l'armée n'a pas le droit d'intervenir dans des affaires judiciaires ou politiques -, on assiste en effet au déploiement d'un dispositif grâce auquel l'appareil d'État tente d'attacher l'usage exceptionnel de la force à un acte juridique. C'est le sens de la scène lors de laquelle Leo et Fitzwallace informent officiellement («submit to you for notification ») les politiciens qu'ils réunissent autour d'eux qu'une action militaire va avoir lieu sans en donner les détails. 
Approuvée par les chefs des services secrets et par les autorités militaires, Leo précise que durant cette "covert operation ", "The President's rescinding his own Executive Order ", ce qui atteste d'une forme de séparation au sommet même de l'exécutif, alors que c'est le Président qui va donner le feu vert. Si je comprends bien cette séquence - pas tout à fait claire quand on n'est pas familier du fonctionnement des institutions états-uniennes -, cela signifie qu'un artifice légal permet à Bartlet de décider sans être considéré, d'un point de vue légal, comme l'auteur de la décision, confirmant ainsi qu'on se trouve dans un régime d'exception.

28 À la fin de l'épisode, qui coïncide avec la fin de la saison, Bartlet doit ainsi se résoudre à donner son accord - dans une configuration tout à fait dissemblable à celle qui le voit trancher entre les options que lui soumettent avec respect ses conseillers dans les affaires courantes. Il a beau réitérer ses déclarations initiales - preuve que sa position n'a pas bougé d'un iota - «I want him tried » ou le catégorique «It's just wrong. It's absolutely wrong ", la réponse de Leo («I know, but you have to do it anyway ») confirme que le pouvoir de décision du Président est enserré dans les rets des scénarios préparés par les militaires, qui s'imposent à lui, comme la carte du prestidigitateur à celui qui croit faussement qu'il a le choix.

Bartlet est-il libre de décider à cet instant ? Dans le fond, c'est peut-être indécidable, tant il est malaisé de déterminer le pouvoir réel dont il dispose lorsque la machine de guerre lancée. Quoi qu'il en soit de la réalité de cette liberté, l'angoisse qui en accompagne l'exercice bouleverse véritablement celui à qui il incombe de prendre une telle décision. L'inventivité des scénaristes est d'ailleurs d'ajouter à ce qu'on pourrait appeler une elliptique des pouvoirs constituée par les deux centres de gravité, formés par le Bureau ovale et la salle de crise, un troisième point de focalisation à partir duquel observer le phénomène de la délibération.

Il est en effet donné à voir les tourments de l'âme qui enferment le Leader of the free world au point de l'obliger à consulter le psychanalyste Stanley Keyworth, espérant, suppose-ton, que ce dernier lui donne une "clé valable " pour sortir de l'impasse. En vérité, la séance d'analyse en face-à-face se déroulant au début de l'épisode n'apporte guère de réconfort à un Bartlet tenu au secret et qui, aux yeux de la loi - non pas de l'inconscient mais de la Cour Suprême - rendrait complice celui qui en entendrait la divulgation. Le régime du droit, à la fois impuissant face à la force mais continuellement mobilisé pour l'encadrer, semble ici obérer toute possibilité de libération par la parole ce qui, dans un final époustouflant, fait pleinement entrer le personnage de Bartlet dans la tragédie.

Les brèves associations de pensées évoquées devant Keyworth nous ont appris que le Président s'est réveillé en chantant une chanson qu'il va entendre lors d'une représentation théâtrale intitulée The War of the Roses à laquelle il doit assister le soir même. Le refrain « and victorious in war shall be made glorious in peace » rappelé par Bartlet révèle à la fois le conflit qui le mine et son secret espoir de voir se réaliser l'adage Si vis pacem, para bellum. Tout comme Hamlet ou EEdipe Roi - autres personnages confrontés à la question de la décision - permettent à Freud d'illustrer et de conceptualiser la dynamique des conflits inconscients, les images de théâtre qui s'intercalent avec celles de la mise à mort de l'ennemi dans le non-lieu des Bermudes, donnent à voir un autre espace, tragique, à l'intérieur duquel se déroule le processus de délibération. Il n'y a ici plus de conseillers, et seul transparaît la solitude du souverain dont le pouvoir est si grand qu'il n'est jamais, en tant que personne, à la hauteur de l'image que l'histoire ou la fiction toujours intimement liées - conservent de lui. 
32 À côté de l'antagonisme entre les temporalités propres au droit et à la force, The West Wing parvient également à montrer comment l'incarnation du pouvoir repose sur une sorte de hors temps fictionnel ou fantasmatique à l'intérieur duquel les gouvernants doivent, génération après génération, porter le poids de l'expérience de la prise de décision, expérience d'autant plus douloureuse que c'est une mise à mort qui est en jeu, à savoir l'incarnation même de l'inéluctable.

33 C'est d'ailleurs lorsque Bartlet est conduit à répondre de ses décisions, quelques épisodes après la mise à mort de Shareef qu'il devient grand, à la hauteur de la fonction qu'il occupe. Comme il l'avait prévu dès le départ, il n'était guère possible qu'une «covert operation » aux implications aussi graves ne soit pas découverte un jour ou l'autre. Ainsi, alors que le personnel de la Maison-Blanche cherche à étouffer l'affaire, le Qumar menace de déclarer que la mort de Shareef n'est pas accidentelle (4.1 et 4.2); même s'il en attribue l'origine à un allié des États-Unis, cette nouvelle replonge le Président dans un conflit qu'il savait latent, le contraignant à endosser la responsabilité et, ce faisant, à retrouver son leadership face aux militaires. Au début de l'épisode pourtant, ce n'est pas gagné. Il semble s'être totalement absenté de la Situation Room où Leo et Fitzwallace reçoivent la conseillère pour la sécurité nationale, Nancy, qui, extrêmement remontée, déclare : «let's recommend to the President we attack». Entre la «recommandation » et «la commande » l'écart semble s'être à nouveau rétréci, ce qui finirait presque par nous convaincre que les militaires continuent à diriger depuis leur forteresse les décisions présidentielles ${ }^{10}$.

Mais les auteurs de The West Wing admirent trop leur démocratie pour que l'on en reste là. Bartlet n'est donc pas seulement représenté au travers de sa solitude, frêle protection contre le coup d'État - les scénaristes se plaisent, en effet, à mettre en scène la façon dont il reprend la situation en main. Pour ce faire, l'interaction est relocalisée dans le Bureau ovale, centralité à partir de laquelle la légitimité du pouvoir du Président peut à nouveau s'affirmer ${ }^{11}$. Alors que Nancy réitère sa " recommandation ", il lui réplique : «I've signed a piece of paper ", mobilisant dans le même temps la légitimité du peuple et la force du droit pour asseoir sa position. Il poursuit en expliquant qu'en cas d'attaque, médiatique ou militaire, provenant de la part du Qumar, il ne se dérobera pas à ses responsabilités, c'està-dire en somme qu'il ira jusqu'au bout du processus décisionnel - même si par un artifice juridique il n'est pas considéré comme ayant pleinement donné l'executive order. Il clôt la discussion en déclarant: "It was my order: and I stand by it ». Cette reprise en main va jusqu'à inverser le fonctionnement courant observé durant toute la série: au lieu d'être appelé à tout bout de champ dans la Situation Room, dans ce cas-là (le seul ?), c'est Bartlet qui donne l'ordre d'y aller ( «et's go ») avant d'être suivi par les militaires.

Le décrochage auquel on a assisté dans la salle de crise semble donc surmonté, et le cadre de la légitimité démocratique à nouveau rétabli. En répondant de ses actes, même les moins glorieux, le Président - lui qui a pourtant tant caché - réaffirme que son pouvoir, c'est-à-dire celui que le peuple lui a confié, ne saurait être spolié par le Pentagone, une institution qui, précisément, aimerait bien ne pas avoir à rendre de comptes. Si le secret a entouré la décision initiale, le processus de divulgation des informations atteste toujours, en fin de parcours, d'une volonté de réaffirmer le droit.

On assiste alors à une autre forme de circulation, qui n'est plus celle des idées élaborées par les conseillers convergeant vers le chef, mais celle par laquelle l'expression de la responsabilité est dirigée vers l'extérieur. La conception de la liberté se révèle alors très singulière puisque même si le Président n'avait pas le choix, il doit assumer sa responsabilité face à un énonciataire indéfini, le peuple, qui, en dernière instance, doit 
assurer le contrôle des décisions prises par l'exécutif. Avant d'examiner selon quelles modalités s'exerce ce contrôle, c'est-à-dire comment est légitimé le pouvoir dans la série, il est intéressant de rappeler que c'est également au sein de la sphère domestique que Bartlet doit endosser la responsabilité de ses décisions.

Nous sommes au début de la cinquième saison dans le domicile personnel dans lequel Jed Bartlet s'est réfugié avec les siens durant l'éprouvant enlèvement de Zoé. En plus d'être morte d'inquiétude, Abbey est furieuse d'apprendre que son mari lui a caché que, naguère, il a ordonné de tuer Shareef. Elle ne peut s'empêcher d'établir un lien de causalité :

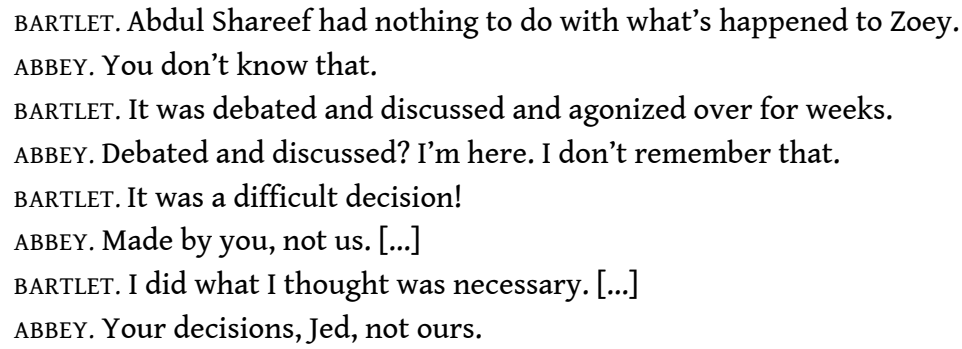

Dans ce passage, on voit se confirmer ce que nous apprenaient déjà les scènes - dans tous les sens du terme - dans lesquelles Abbey reproche à son époux d'avoir décidé de se représenter à l'élection sans l'avoir consultée : parmi les innombrables conseillers qui travaillent au service du Président, l'influence de la First Lady est cruciale, y compris lorsqu'il est question de stratégie politique. Même si la séparation des sphères est nette dans l'organigramme, et que l'espace domestique est disjoint de la Maison-Blanche, il faut donc comprendre que, sauf exception, les décisions les plus importantes donnent lieu à des discussions du Président avec sa femme. En ces occasions, Abbey ne défend pas les mêmes valeurs que Leo et les militaires qui sont à la manœuvre par son entremise ; pourtant son influence s'exerce également dans les interstices du pouvoir légitime même si le statut de l'épouse du Président est mieux codifié aux États-Unis qu'en France.

À l'issue de cette rapide inspection, il apparaît que, même si le Président doit toujours prendre des décisions en son âme et conscience, il ne cesse d'être entouré par des conseillers, plus ou moins occultes, dont le nombre semble se démultiplier sans cesse. L'exercice du pouvoir implique donc une forme d'impureté, de combinaison de force et de droit, d'officiel et d'officieux, dont les scénaristes nous donnent à voir la complexité épisodes après épisode. Au milieu de ce maelström quotidien, c'est paradoxalement la solitude essentielle, constitutionnelle, du Président qu'il faut retenir. Au sein de sa famille, de son administration ou de la salle de crise, Bartlet est le seul à avoir été élu - si on excepte le Vice-Président dont il n'est pas besoin de préciser qu'il reste la plupart du temps en marge des affaires les plus importantes. Si les discours préparés par les hommes de l'ombre donnent parfois l'impression qu'on assiste à une forme de ventriloquie, dans le fond, Bartlet est conçu et se conçoit comme le représentant et le porte-parole du peuple souverain. Fort heureusement, les conseillers étant animés d'un même idéal font qu'il n'est pas tout seul pour remplir cette mission. Dans cette perspective, le personnage de Toby joue un rôle fondamental dont il convient de dire un mot pour saisir par quels procédés la souveraineté du peuple, c'est-à-dire la forme suprême de la décision, est représenté dans The West Wing. 


\section{La société dans l'État : la Constitution comme cadre démocratique pour la délibération}

Même si Toby n'est officiellement que Communications Director, il occupe une place centrale parmi les conseillers de l'Aile ouest, en vérité beaucoup plus importante que celle de Josh, Sam ou C.J., personnages dont il constitue une sorte de synthèse. Il possède l'intelligence stratégique de Josh et partage avec C.J., qu'il a recrutée, une forme de sensibilité idéaliste dès lors que la défense des droits civiques et des libertés fondamentales est menacée. Son originalité est de combiner cet ethos militant avec une connaissance approfondie du droit, qui n'a rien à envier à celle de Sam, surtout pour ce qui concerne la Constitution des États-Unis. C'est en effet dans le droit constitutionnel que Toby excelle, relisant sans cesse ce texte fondateur - jusqu'à y trouver une faute de ponctuation! - en le reliant à la réalité mobile du monde contemporain qui se déploie sous ses yeux.

41 Pour interpréter le monde, Toby dispose d'une grande finesse psychologique qui lui permet de découvrir les pensées des autres en observant simplement quelques signes, comme dans l'épisode dans lequel il comprend que le Vice-Président prépare sa candidature pour les élections présidentielle car il « sait quelque chose qu'il ne sait pas » (la maladie de Bartlet). Pas besoin de faire circuler les idées ici : il suffit d'avoir les prémisses pour, logiquement, comprendre les intentions d'autrui. Avec sa balle de baseball qu'il fait rebondir sur les murs de son bureau, ou le geste de sa main caressant son crâne, les auteurs de The West Wing nous montrent Toby comme un personnage qui ne cesse de réfléchir et cherche à décrypter ainsi les événements du monde.

Dans une telle entreprise, le droit et la Constitution lui servent également de boussole, sans doute parce qu'il y trouve un moyen de concilier son aspiration pour la liberté individuelle avec un ordre rationnel établissant des garde-fous pérennes qui empêchent les décisions des gouvernants de devenir arbitraires. Lors de l'épisode dans lequel il aide les nouveaux dirigeants d'un pays Balte à rédiger leur Constitution, il fait d'ailleurs sentir à ces derniers l'importance d'un tel document pour les destinées d'un peuple : la moindre faille dans sa rédaction peut avoir des conséquences néfastes dans le futur. À cette occasion, Toby souligne l'imperfection de la Constitution de son pays : à ses yeux elle ne serait pas capable d'empêcher la prise de pouvoir par un dictateur. Alors que le document fondateur devrait nécessairement empêcher tout changement de régime par la violence, il semble que ce ne soit que pour des raisons contingentes que les États-Uniens ont eu la chance de ne pas avoir connu de période aussi dramatique.

Pour de multiples raisons, Toby semble hanté par la crainte d'un coup d'État: on en trouve un parfait exemple au moment où il découvre que le Président a menti sur sa santé (2.18). Encore abasourdi par la nouvelle d'une telle occultation, et meurtri de ne pas avoir été mis dans la confidence, Toby n'en continue pas moins à déployer son pouvoir analytique afin de suivre toutes les implications liées à cette sclérose en plaques. Par-delà les questions affectives ou strictement politiques, conformément à sa grille de lecture, c'est sans surprise qu'il en vient à réfléchir en spécialiste de la Constitution aux conséquences de l'incapacité à pouvoir gouverner pour des raisons de santé1 ${ }^{12}$. Dans l'épisode 2. 18, c'est précisément pour des raisons juridiques que Toby en vient à conclure qu'il y a eu un coup d'État, comme on l'entend dans le dialogue suivant : 
BARTLET. [sarcastic] Toby's concerned that the peaceful solution I brokered last year in Kashmir was the result of a drug-induced haze. [...]

LEO. [to Toby] I was there with him. So was Fitz. So was Cashman, Hutchinson, Berryhill... [...]

TOBY. None of you were elected!

BARTLET. I was elected, they were appointed. [points at Leo] The Vice President was elected. He has the constitutional authority to assume my...

тову. Not last May he didn't. [rises] He didn't last May when you were under general anesthesia.

BARTLET. That's because I never signed the letter and I don't think I got shot because I got MS.

TOBY. I don't think you did either, sir. I meant that during a night of extreme chaos and fear, when we didn't yet know if we'd been the victims of domestic or foreign terrorism or even an act of war, there was uncertainty as to who was giving the National Security orders, and that was because you never signed a letter! [...] [angry] The Commander-In-Chief had just been attacked. He was under a general anesthetic. [...] The National Security Advisor and Secretary of State didn't know who they were taking their orders from! I wasn't in the Situation Room that night but I'll bet all the money in my pockets against all the money in your pockets that it was Leo... whom no one elected! For 90 minutes that night there was a coup d'État in this country. assiste à la fin de la série à la progressive décomposition des relations, voire à l'irruption de conflits insolubles entre les protagonistes. Dans un épisode, une rivalité demeurée latente entre Josh et Toby se déclare à mesure que se pose la question de la succession de Bartlet: ils en viennent même aux mains (6.16). À part cet épisode, le spectateur est surtout navré d'avoir à être témoin des conséquences de la fuite organisée par Toby 
lorsqu'il apprend qu'un programme spatial contrevenant aux traités internationaux encore le droit - est conduit dans le plus grand secret par les militaires (7.5).

La confession devant C.J., puis le renvoi par la Maison-Blanche de celui qui aura toujours été un fidèle parmi les fidèles, ont quelque chose de profondément injuste ; d'autant plus que Toby, emporté par son côté belle âme, se refuse à mentir pour éviter la prison. Pourtant, même si cette fin confère une tonalité un peu amère aux derniers épisodes nouvelle preuve des qualités des scénaristes évitant le happy end -, elle était inévitable. Non pas simplement parce que, comme le déclare Bartlet à Toby, ce dernier aurait toujours méprisé son supérieur hiérarchique ; par-delà la dimension psychologique, c'est en effet l'organisation même du pouvoir à l'intérieur de la Maison-Blanche qui recèle une tension entre des centres de décision aux principes irréductibles les uns aux autres, et même dans certains cas incompatibles.

En ne gardant que l'essentiel, le schéma d'une telle organisation pourrait être représenté par un triangle au sommet duquel Bartlet aurait à composer avec les forces antagonistes et des régimes de légitimation divergents mobilisés par Leo d'un côté et par Toby de l'autre. Dans ce cadre, l'opprobre qui s'abat sur Toby, lorsqu'il est renvoyé de la MaisonBlanche, n'empêche pas que l'ultime décision du Président consiste à lui accorder une grâce afin de lui éviter la prison. On pourrait suggérer qu'en faisant avec réticence un tel choix, il rencontre une configuration ressemblant à celle qui le contraint à donner l'ordre de tuer Shareef : dans les deux cas, Bartlet se sent contraint de faire ce qu'il ne veut pas faire. Il y a dans cette possibilité offerte au Président d'accorder sa grâce quelque chose de profondément arbitraire et, à certains égards, d'antidémocratique.

Pourtant, les choses se révèlent bien différentes du drame vécu lorsqu'il faut se rallier à l'option élaborée par les militaires. Au contraire, bien qu'accordée «avec mauvaise grâce ", si l'on peut dire, la protection de Toby signifie qu'il n'a pas totalement eu tort de faire circuler des informations comme il l'a fait. Bartlet ne peut évidemment pas le dire ainsi, pas plus qu'il ne peut donner son assentiment aux militaires sans marquer dans le même temps son désaccord. Une phrase un peu alambiquée prononcée au moment du renvoi de Toby illustre parfaitement cette ambivalence :

BARTLET. When you walk out of here, there'll be people out there, perhaps a great many, who'll think of you as a hero. I just don't for a moment want you thinking I'll be one of them.

Ulcéré par ce qu'il considère être une trahison, personnelle et professionnelle, le Président entend affirmer haut et fort sa désapprobation, d'autant plus que la dernière entrevue avec son conseiller se déroule sous le regard de Babish. Mais, dans une sorte d'éloge paradoxal, Bartlet exprime aussi qu'à «l'extérieur» le traître pourra être considéré comme un héros. Cette reconnaissance, à mot couvert, d'une autre interprétation de l'acte de divulgation en direction de la société civile, prouve que le Président a conscience d'un tiraillement entre deux formes de légitimité qu'il parvient à faire tenir ensemble par le biais d'un nouvel artifice juridique : en signant finalement la grâce il peut, sans contradiction, déclarer un homme coupable tout en lui évitant d'avoir à subir la peine.

51 En fin de parcours, c'est donc bien la légitimité démocratique, relayée par l'acte de signature qui reprend ses droits au sein du Bureau ovale. En nous montrant un livre de Foucault sur une étagère, les auteurs affirment d'ailleurs sans ambiguïté leur position, presque un credo : le peuple doit toujours être présent à l'intérieur du lieu de pouvoir. Ce n'est pas donc pas «la Société contre l'État» - pour reprendre le titre de l'ouvrage de P. 
Clastre - qui est mise en scène, mais la société dans l'État. Cela se fait sans idéalisme ; au contraire le génie des auteurs est de nous donner à voir, épisode après épisode, le fonctionnement hybride de l'édifice du pouvoir, tiraillé par des principes divergents, et l'impureté de toutes les décisions que prend le Président des États-Unis.

\section{Conclusion}

52 De ce qui précède, on peut retenir que The West Wing nous donne à voir au moins trois formes de délibération auxquelles correspondent des types de circulations d'idées spécifiques. Pour les affaires courantes, les délibérations du Président interviennent après qu'un processus de sélection a permis aux idées émanant de la société de converger vers le Bureau ovale. À l'opposé de ce processus qui exige temps et médiation, les décisions prises dans la Situation Room se caractérisent par leur immédiateté, chaque nouvelle information conduisant presque instantanément à une nouvelle réaction. Dans ce contexte, l'autorité subit une forme de décentrement puisque ce n'est pas véritablement le Commandant en chef qui décide, mais ceux qui font l'expérience, génération après génération, du champ de bataille. Lorsque les auteurs insistent sur la conformité de certaines décisions avec le droit, c'est une autre histoire qu'ils montrent : dans ce cadre, chaque décision doit être conçue dans son lien avec les décisions fondatrices prises par le peuple qui s'incarnent dans la Constitution. Conformément à cette logique, comme Toby en assume le risque, il est important qu'un mouvement de rétrocession dans la circulation des idées s'effectue, afin que le peuple ne soit pas privé de la connaissance des actions des gouvernants.

Par conséquent, la délibération combine toujours de l'hétérogène. Au niveau des principes, puisque chacun des conseillers qui participe aux décisions du Président le fait à partir d'un dispositif de captation du réel et d'un univers axiologique qui lui sont propres, obligeant les protagonistes - en premier lieu Bartlet - à opérer un continuel travail d'agencement pour maintenir ensemble des réalités distinctes. En se contentant de repérer une opposition entre le pouvoir civil et le pouvoir militaire, on perdrait toute la subtilité de la série qui tient précisément dans sa capacité de ne pas aborder le réel à partir de dichotomies. Si on a vu qu'il existe une continuelle tension entre la force et le droit - il serait d'ailleurs plus juste de parler d'un processus dans lequel le droit cherche à englober l'usage de la force qui tente de se déployer hors de son règne -, ils se révèlent évidemment nécessaires, l'un comme l'autre, à l'exercice plein du pouvoir.

Les États-Unis possèdent d'ailleurs une manière très spécifique de chérir ces deux maitres, au point d'en faire les objets d'une construction mythologique, comme Hollywood en donne souvent l'exemple, en particulier dans les westerns. Un parallèle intéressant peut d'ailleurs être établi avec The Man who Shot Liberty Valance (1962), chefd'œuvre de John Ford, dans lequel le meurtre fondateur est apparemment commis par James Stewart jouant le rôle de l'avocat pacifiste qui deviendra sénateur, alors qu'en vérité la responsabilité revient au valeureux cowboy joué par John Wayne qui tue Liberty Valance depuis une cachette. On est encore en plein feu d'artifice, le dispositif filmique et narratif montrant que, sous un certain point de vue, il existe une disjonction entre le droit et la force et que celui qui briguera les suffrages jusqu'à devenir sénateur n'a en réalité pas de sang sur les mains. Bref, il faut un pouvoir fort, mais la responsabilité morale des représentants du peuple ne doit pas être trop entamée. C'est une histoire un peu similaire, en plus développée, que nous raconte The West Wing, en montrant que non 
seulement il existe différentes manières d'agencer des principes antagonistes, mais aussi que, par diverses formes de construction, le pouvoir parvient à rendre aussi peu visible que possible la suture entre des éléments hétérogènes, procédés courant dans la pensée mythique.

Pour ne pas terminer sur un happy end trop laudatif, je soulignerai qu'en dépit de la valorisation de la démocratie, les auteurs de The West Wing ne laissent pas d'être fascinés par une forme d'élitisme laissant peu de place au demos, précisément. L'importance de la mission confiée aux gouvernants exige indéniablement un niveau d'excellence académique dans le recrutement des conseillers qui proviennent tous des meilleures universités, on ne cesse de nous le rappeler. Mais, il n'était sans doute pas nécessaire que cette admiration pour une forme d'entre soi de classe se fasse au détriment du peuple. Deleuze a bien montré par quels procédés narratifs et filmiques les cinémas d'Eisenstein et de Vidor offrent des représentations distinctes du peuple, chacune conforme à l'idéologie politique de leur nation.

Dans The West Wing, on est étonné de constater comment le sentiment patriotique mâtiné de sens du devoir des principaux protagonistes entre en contradiction avec le traitement souvent dépréciatif de ceux qui vivent hors de la Maison-Blanche, comme le montrent les deux épisodes dans lequel Josh, Tobby et Donna sont comme perdus dans leur propre pays. C'est souvent sous l'angle sentimental que le peuple fait irruption, comme une multitude émouvante ou émue, mais l'inventivité politique du peuple ne semble pas mériter d'être mise en avant, comme elle l'est chez Capra par exemple, un autre grand producteur de mythes. Le canular organisé pour que des faux électeurs fassent croire à Josh qu'ils n'ont pas su exprimer correctement leur vote apparait de ce point de vue comme une sorte de retour du refoulé: au moment même où il devrait agir de façon souveraine, ce peuple tant respecté prend le visage de l'incompétence et de l'ignorance !

Tout se passe comme si, en montrant comment les décisions politiques parvenaient toujours à homogénéiser des positions et des principes hétérogènes, les créateurs de la série n'avaient pu faire autrement que de prendre acte - par réalisme ou par fascination pour le pouvoir - de la séparation radicale entre les gouvernants et les électeurs. Cette séparation qui mine de l'intérieur la construction démocratique idéalisée de mille manières semble quant à elle difficile à résorber. De l'autre côté de la frontière, en dépit des inégalités existant dans les communautés indiennes du sud du Mexique, cette séparation n'est pas encore aussi nette, puisque à une échelle plus réduite - quelques milliers d'habitants -, il est plus aisé de maintenir une rotation et une permutation dans les positions sociales. L'enjeu, tant pour la politique que pour les arts visuels, est alors de déterminer s'il est possible de faire subir à une organisation politique démocratique une variation quantitative sans produire, à partir d'un certain seuil, des effets qualitatifs négatifs, conduisant éventuellement à des changements de régime. En somme, comme le faisait Deleuze en citant Paul Klee, il convient de se demander comment on peut représenter le peuple qui manque. 


\section{BIBLIOGRAPHIE}

BOLTANSKI Luc et Laurent Thévenot, De la justification. Les économies de la grandeur, Paris, Gallimard, 1991.

BOLTANSKI Luc, De la critique. Précis de sociologie de l'émancipation, Paris, Gallimard, 2009.

DELEUZE Gilles, L'Image-Temps. Cinéma 2. Paris, Éditions de Minuit, 1985.

LATOUR Bruno, « Le fantôme de l'esprit public. Des illusions de la démocratie aux réalités de ses apparitions ", préface à Le Public Fantôme, traduction française de The Phantom Public, par Walter Lippmann (traduction Laurence Decréau), Paris, Éditions Demopolis, 2008, pp. 3-49.

LATOUR Bruno, La fabrique du droit. Une ethnographie du Conseil d'État, Paris, La Découverte, 2002.

LATOUR Bruno, Enquête sur les modes d'existence. Une anthropologie des Modernes, Paris, La

Découverte, 2012.

HOBBES Thomas, Leviathan: Or the Matter, Forme, and Power of a Common-Wealth Ecclesiasticall and Civill, éd. Ian Shapiro, New Haven, Yale University Press, 2010 [1651].

PITROU Perig, «Justice et agentivité distribuée chez les Mixe de Oaxaca (Mexique). Approche cosmopolitique ", Ateliers d'anthropologie, $\mathrm{n}^{\circ} 39,2013$. Perig Pitrou, « Le pouvoir entre exposition et occultation. Analyse de rituels politiques dans la Sierra Mixe de Oaxaca, Mexico ", Cahiers d'anthropologie sociale, $\mathrm{n}^{\circ} 11,2015$.

\section{NOTES}

1. Conformément à l'usage, les ethnonymes provenant de langue étrangères sont invariables tant qu'ils n'ont pas été francisés.

2. Perig Pitrou, «Justice et agentivité distribuée chez les Mixe de Oaxaca (Mexique). Approche cosmopolitique ", Ateliers d'anthropologie, $\mathrm{n}^{\circ}$ 39, 2013. Perig Pitrou, «Le pouvoir entre exposition et occultation. Analyse de rituels politiques dans la Sierra Mixe de Oaxaca, Mexico ", Cahiers d'anthropologie sociale, $\mathrm{n}^{\circ}$ 11, 2015.

3. Bruno Latour, « Le fantôme de l'esprit public. Des illusions de la démocratie aux réalités de ses apparitions ", préface à Le Public Fantôme, traduction française de The Phantom Public, par Walter Lippmann (traduction Laurence Decréau), Paris, Éditions Demopolis, 2008, pp. 3-49.

4. Luc Boltanski, De la critique. Précis de sociologie de l'émancipation, Paris, Gallimard, 2009.

5. Bruno Latour, Enquête sur les modes d'existence. Une anthropologie des Modernes, Paris, La Découverte, 2012.

6. Pour approfondir cette analyse concernant le rôle des objets, on pourrait également s'inspirer des passages consacrés par Gilles Deleuze au cinéma d'Alfred Hitchcock. Gilles Deleuze, L'ImageTemps. Cinéma 2. Paris, Éditions de Minuit, 1985.

7. Luc Boltanski et Laurent Thévenot, De la justification. Les économies de la grandeur, Paris, Gallimard, 1991.

8. Bruno Latour, La fabrique du droit. Une ethnographie du Conseil d'État, Paris, La Découverte, 2002.

9. Thomas Hobbes, Leviathan: Or the Matter, Forme, and Power of a Common-Wealth Ecclesiasticall and Civill, éd. Ian Shapiro, New Haven, Yale University Press, 2010 [1651]. 
10. On remarquera que c'est précisément l'épisode dans lequel Josh, Toby et Donna sont "perdus » au fin fond du pays « réel », ce qui redouble en quelque sorte l'idée d'une vacance du pouvoir.

11. La puissance du lieu est d'ailleurs telle qu'on découvre qu'un ancien Président, nostalgique de ses années à la Maison-Blanche, fait construire une réplique du Bureau ovale dans sa demeure (5.10). Mais sans la légitimation par les urnes, ce symbole de pouvoir apparaît évidemment comme une fixation pathétique à un passé révolu.

12. On notera à ce propos que la question de la fragilité du corps du souverain, mais aussi de l'exercice de sa faculté judiciaire, ressurgit à plusieurs reprises. Alors que la théorie des « deux corps du roi " étudiée par Kantorowicz visait à assurer la permanence de la royauté, les scénaristes de The West Wing soulignent combien, du fait de son incarnation dans un corps, le processus décisionnel risque toujours d'être biaisé, soit du fait du déclin des capacités cognitives, soit à cause d'un état émotionnel trop intense - ce qui conduit Bartlet, par un autre artifice juridique, à transférer son pouvoir lorsque sa fille est enlevée (5.1 et 5.2).

\section{RÉSUMÉS}

À travers l'analyse de quelques séquences de The West Wing se déroulant dans la Situation Room, je souhaite réfléchir à la façon dont sont mis en scène les processus de délibération lorsque des conflits graves, mettant en danger la sécurité intérieure ou extérieure, doivent être traités par le pouvoir exécutif. Comment se manifestent les doutes et les cas de conscience? Quels sont les dispositifs - techniques, interactionnels, rituels - grâce auxquels ils sont surmontés ? Quels sont les agents mobilisés pour parvenir à un tel résultat? Comment les idées circulent-elles le long de la hiérarchie et, en retour, comment se distribuent les responsabilités, une fois les décisions prises? Tels seront quelques uns des problèmes abordés. Plus largement, il s'agira de faire contraster la spécificité des prises de décision guidées par l'expérience des militaires avec les contextes, plus quotidiens, dans lesquels les suggestions des conseillers convergent vers le Président afin de l'orienter dans sa réflexion. Dans ce contexte de tension, voire d'antagonisme, le rôle central joué par le Droit et le Constitution sera mis en évidence.

By analyzing a few sequences from The West Wing taking place in the Situation Room, I will analyze the representation of deliberation whenever the executive power is facing serious conflicts endangering interior or exterior security. How are doubts and crises of concience represented? What devices, be they technical, interactional or ritual, are used to overcome them? How ideas circulate within the hierarchy, and how responsibilities are distributed, once decisions have been made - these are the main issues we will focus on. We will lay emphasis on the contrast between the specificity of decisions guided by the experience of the military and the more trivial context in which advisers' suggestions attempt to guide the President's reflection. In this tense, sometimes antagonistic context, we will explain the essential role played by the Law and the Constitution. 
INDEX

Mots-clés : À la Maison Blanche, West Wing (The), série politique, délibération, responsabilité politique, président des États-Unis

Keywords : West Wing (The), political series, déliberation, political responsibility, American Presidency

\section{AUTEUR}

\section{PERIG PITROU}

Perig Pitrou est anthropologue au CNRS, rattaché au Laboratoire d'anthropologie sociale du Collège de France. Dans le cadre d'un travail doctoral, il a réalisé une mission ethnographique de deux ans parmi des populations amérindiennes de l'État de Oaxaca au Mexique et a été pensionnaire de la Casa de Velázquez et du CEMCA, puis chercheur au musée du quai Branly. Ses recherches portent sur la ritualité, les justices traditionnelles et sur les représentations, conceptuelles et figuratives, de la vie. Il a dirigé l'ouvrage La noción de vida en Mesoamérica (CEMCA-UNAM) et il est l'auteur d'une monographie intitulée Le chemin et le champ. Parcours rituel et sacrifice chez les Mixe de Oaxaca, Mexique (Société d'ethnologie de Nanterre).

Perig Pitrou is an anthropoligist at the CNRS, from the Social Anthropology Unit at the Collège de France. For his doctoral research, he led a 2-year ethnographic survey within American Indian populations of the Oaxaca state in Mexico, and was a member of the Casa Velazquez and the CEMCA, then a researcher for the Musée du Quai Branly. His research focuses on rites, traditional justice and conceptual and figurative representations of life. He directed La noción de vida en Mesoamérica (CEMCA-UNAM)and wrote a book entitled Le chemin et le champ. Parcours rituel et sacrifice chez les Mixe de Oaxaca, Mexique (Société d'ethnologie de Nanterre). 